\title{
DINÂMICA DOS ISOTÓPICOS ESTÁVEIS DO CARBONO E OXIGÊNIO NO ESTUÁRIO DO RIO PARAGUAÇU - BAHIA
}

\author{
LUIZ CARLOS PINA
}

Laboratorio de Fisica Nuclear Aplicada, Instituto de Fisica, Programa de Pesquisa e Pos-Gradụaça em Geofisica da Universidade Federal da Bahia

\begin{abstract}
Measurements of $\$ C 13$ and $\$ O 18$ have been made in water samples and carbonate sediments from the Paraguaçu estua$r y$. with to a precision of $\times 0,1 \%$.

Water samples wete collected in the complete salinity range from $0 \%$, to $35 \%$. with the fresh water having the lowest $018 / 016$ ratios of $-1,3 \%$. Alinear correlations exists between salinity and the oxygen isotopic composition suggesting

that $\mathrm{O} / 8 / \mathrm{O} / 6$ behaves as a conservative property during the mixing of fresh water with sea water.

With respect to the carbonate sediments, there exists a correlation between 8018 and $\$ C 13$, indicating the need of $\triangle \mathrm{C} / .3$ measurements when 8018 is being used as a temperature indicator. Since the carbonate shells are derived from soluble HCO.3, it is shown the possibility of using \$O18 as an indicator of the mean salinity at a given locality.
\end{abstract}

\section{INTRODUÇÃO}

A Baía de Todos as Santos, situada perto de Salvador, Brasil, cobrindo uma área de aproximadamente $800 \mathrm{~km} 2$, fornece uma excelente oportunidade para o estudo de processus ambientais.

A grande extensão da abertura da Baía, situada entre o farol da Barra e alocalidade de Mar Grande (Itha de Itaparica) faz com que o seu interior apresente essencialmente a mesma salinidade do mar aberto, exceto nas regióes próximas à foz do rio Paraguaçu (Fig. 1).

A vazão média do rio Paraguaçu, excetuando o período de chuvas, não é muito grande, apresentando um valor de carca de $45 \mathrm{~m} 3 / \mathrm{seg}$. (C.P.R.M., 1975). Dependendo do período de amostragem, a salinidade na foz do rio pode variar entre $35 \%$ e e $7 \%$ o, em função das marés e da vazão.

Para cada local da zona transicional de mistura, a salinidade varia continuamente durante o ano. Mensalmente perfís de salinidades do rio tem sido efetuados (Peixinho, 1974), musıstrando grandes flutuações na região. Por outro lado, pode-se mosirar que geralmente uma variação na salinidade corresponde a uma variação no teor em O18 dessas águas (Epslein e Mayeda, 1953).

A zuna de mistura escolhida para a realização deste trabaIho está licalizada entre a Ponta do Alambique e Coqueiros (Fig. 1).

Amostras de águas coletadas na zona de mistura, têm por objetivo tentar estabelecer uma relação salinidade - razão iscrípica O18/O16 para o sistema constituido pelo rio Paraguaçu. Por outro lado, tenta-se mostrar que a composição iscrópica do oxigênio das conchas, pode fornecer salinidades médias para cada localidade durante o crescimento do molusco. Já que a variação de crescimento sazonal é pequena e estatisticamente representa a média de flutuações da maré durante 0 ano, esta salinidade obtida a partir da razão O18/O16 pode ser considerada como correspondendo a salinidade média anual.

Neste trabalho tentou-se também verificar se os organisnus carbonatados foram formados ou não em equilíbrio isolópico com o meio ambiente.

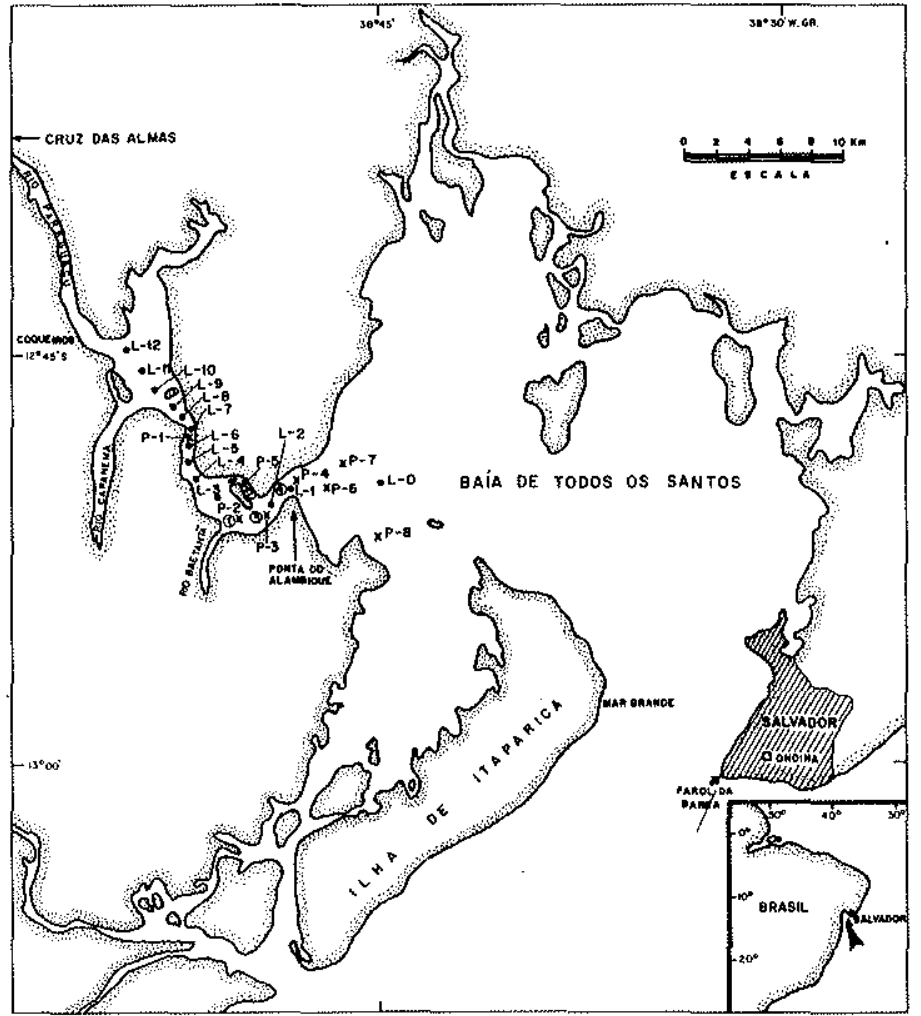

Figura 1 - Estuário do rio Poroguaçu. Os pontos indicam as locais onde as amostros de óguo foram coletados os $\{x\}$ as amostras de conchas carbonatadas.

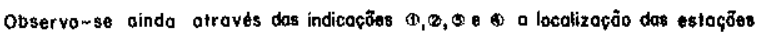
de caletas de aqua realizodos por Peixinho $\{1974\}$. 


\section{MÉTODOS EXPERIMENTAIS}

Em março de 1974, amostras de água foram coletadas na Baía de Todos os Santos e no rio Paraguaçu nos pontos indicados na Figura 1.

O método utilizado para preparacão do $\mathrm{CO} 2$ para análise do oxigênio dessas águas foi descrito por Epstein e Mayeda (1953) e Dansgaard (1961), e consiste em equilibrar isotopicamente a água com o dióxido de carbono que será utilizado para medir a razão isolópica O 18/O 16.

As amostras de conchas carbonatadas foram cedidas pelo Prof. Arno Brichta do Instituto de Geociências da UFBa e são provenientes de coletas da superfície do fundo da Baía, nos pontos indicados na Figura 1. Apesar de não ter sido pussível delerminar as espécies do ponto de vista biológico e consequentemente os hábitos de vida, a constância da distribuição com que estes organismos foram encontrados nas várias colelas efetuadas em diversas épocas do ano sugerem que se trala de espécies que praticamente se desenvolveram in situ, o que elimina, pelo menos em princípio, as dificuldades que poderiam surgir se houvesse uma migração importante destes organismos. Para as análises, foram selecionadas conchas bem conservadas, sem fraturas e de unia mesma textura, provenientes de organismos recentes, guardando ainda suas características originais. As amostras furam então lavadas com água destilada e atacadas com $\mathrm{H} 3$ PO 4 a $100 \%$ sob vácuo (Mook, 1968; Epstein et al., 1953). O CO 2 resultante foi separado, e após purificação, as razões $\mathrm{O} 18$ / O 16 e C 13/C 12 foram medidas através de um espectrîmetro de massa Nuclide 6 - 60 RMS de duplo coletor.

A determinação da salinidade das águas superficiais foi feita por titulação, segundo um método adotado por Peixinho (1974).

Todos os dados isolópicos foram expressos na notação usual $\&(\%$.$) , definido como:$

$$
\$(\% .)=\text { RA - RP } \times 103 \text { onde RP }
$$

$\mathrm{R} A=\mathrm{A} / \mathrm{A}=$ razão isolópica da amostra e $\mathrm{A}=$ isótopo raro $\quad A=$ isólopo abundante

$\mathbf{R} P=A / A=$ razão isotópica do padrão.

$\delta=\$ A=$ desvio relativo da razão isotópica da amostra com respeilo ao padrão, enquanto que as medidas foram feitas usando um padrão secundário calibrado com respeito aos padrões internacionais PDB e SMOW. Correções e estudos de reprodutibilidade foram realizados segundo método desenvolvido por Campos (1976).

\section{DISCUSSÃO DOS RESULTADOS}

\section{Composição Isotópica do Oxigênio nas Āguas}

As análises das amostras de água apresentam valores isotópicos do oxigênio expressos em \&O 18 compreendidos entre $-4,3 \%$. e $0,0 \%$. para pontos de salinidade $0 \%$. e $35 \%$. respectivamente. Valores mais altos no teor em $\mathrm{O} 18 \mathrm{da}$ água são encontrados em pontos de maiores salinidades (Tabela 1), confirmando a variação no teor em $\mathrm{O} 18$ das águas para unıa variação na salinidade já verifícada por Epstein e Mayeda (1953).

Na região en estudo, o rio Paraguaçu é o principal responsável pelo influxo de água doce na Baía, decrescendo o valor isclópico do oxigênio dessas águas em até $-4,3 \%$.

O gráfico construido na Figura 2 mostra uma boa correlaçãuentre salinidade e composição isotópica do oxigênio, com cueficiente de correlação igual a 0,996 indicando que esses resulladus são compatíveis com uma mistura considerada comservativa do ponto de vista isotópico, entre a água do rio Paraguaçu c a água do mar.
TABELA 1

\begin{tabular}{|c|c|c|}
\hline AMOSTRA & $S\left\{\%_{4}\right\}$ & ${ }_{8} 0{ }_{\text {SMOW }}^{8}[\%$ \\
\hline$L-0$ & 35,0 & 0,0 \\
\hline $1-1$ & 21.3 & $.1,5$ \\
\hline$L-?$ & 20,0 & $.1,9$ \\
\hline$L \ldots 3$ & 18,1 & 2,0 \\
\hline$L-4$ & 17,8 & 2,2 \\
\hline$L-5$ & 14,7 & 22 \\
\hline$L-6$ & 13.6 & 25 \\
\hline $1-7$ & 115 & 27 \\
\hline $1-8$ & 6.8 & 34 \\
\hline$L-g$ & 50 & 3,6 \\
\hline$L \cdots 10$ & 1.1 & 40 \\
\hline$[\cdot .11$ & $0,5$. & 4,3 \\
\hline$\lfloor-12$ & 0,0 & 4,3 \\
\hline
\end{tabular}

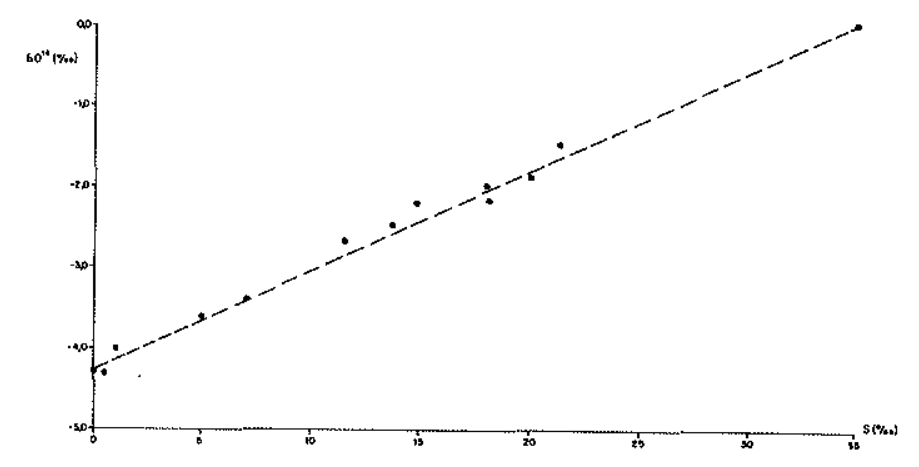

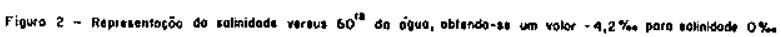

A composição isolópica do oxigênio na foz do rio Paraguaçu é representativa das precipitações na sua bacia de alimenlaçãu. Por outro lado, medidas da razão O 18/0 16 efetuadas enı águas de chuvas amostradas nas regiões de Salvador, Cruz das Almas e de Lençóis, esta última bem próxima das cabeceiras do rio Paraguaçu, mostram valores médios homogêneops para as precipitaçoes que ocorrem em boa parte do Estado da Bahia (SO $18--3,5 \%$. (SMOW), (Tabela 2, Azevedo, 1975). Entretanto, a amostra L-12, correspondendo a uma salinidade igual a $0,0 \%$, apresenta razão isotópica do oxigênio mais negativa do que a média para as precipitações nessa área. Este resultado sugere que esse valor seria devido à preponderância de isótopos mais leves nos períodos de naiores precipitações.

\begin{tabular}{|c|c|}
\hline \multicolumn{2}{|c|}{$\begin{array}{c}\text { TABELA } 2 \\
\text { Resultados dos valores Isotóplcos médlos anuals do } \\
\text { oxigênio em amostras de águas de chuva, segundo } \\
\text { Azevedo (1975) }\end{array}$} \\
\hline $\begin{array}{l}\text { AMOSTRAS } \\
\text { IEslaç̃est }\end{array}$ & SO ${ }_{S M O W}^{18}[\%$ \\
\hline Ondina & 3,7 \\
\hline Cruz das Aimas & 3,3 \\
\hline Lençois & 3,5 \\
\hline
\end{tabular}

COMPORTAMENTO DOS ISOTTOPOS DO CARBONO

E OXIGENIO EM CARBONATOS BIOGENICOS

Os resultados das razões C 13/C 12 (PDB) e O 18/O 16 (PDB) oblidas em amostras de carbonatos são apresentados

Revista Brasileira de Geociências - Volume 9- $\mathbf{N}^{0} 4-1979$ 
na Tabela 3. Foi obtida uma correlação linear (com coeficiente igual a 0,991 ), indicando que existe forte correlação de natureza isotópica entre o carbono e o oxigênio durante a formação dos carbonatos biogênicos (Figura 3).

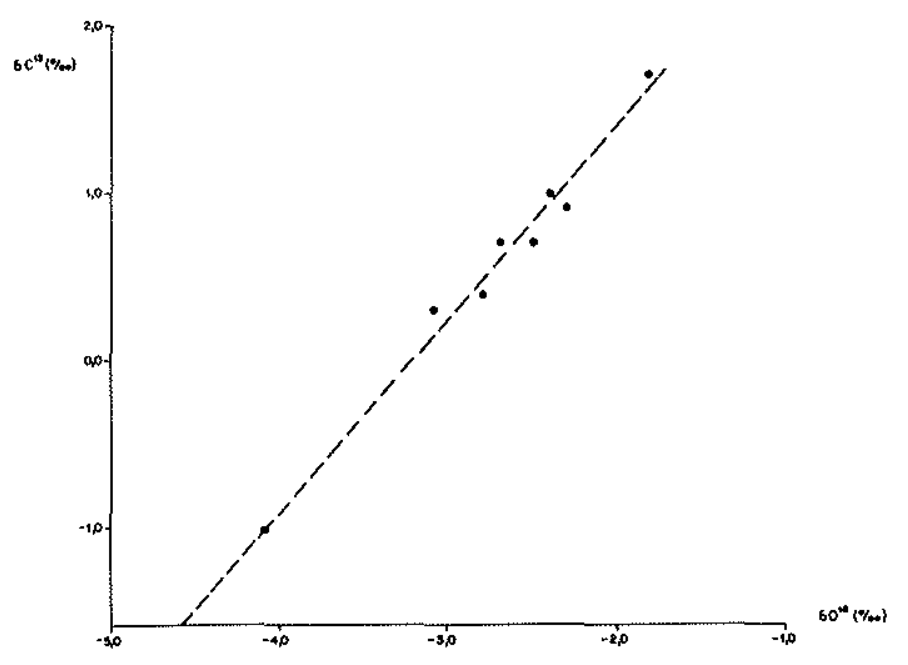

figuzo 3 - Reloçäo entre $6 \mathrm{C}^{43} \cdot 6 \mathrm{O}^{10}$ de conchan torbonatodan.

\section{TABELA 3}

\section{Resultados das análises Isotópleas do carbono $\theta$ oxigênio em amostras de conchas de carbonatos}

\begin{tabular}{|c|c|c|}
\hline AMOSTRAS & $\left.{ }_{8}^{0} 0_{\mathrm{PBB}}^{10} \mathrm{~B}^{10{ }_{1}}\right]$ & ${ }_{\delta} C_{P D B}^{13} \mid[/ \%(m)$ \\
\hline $\mathrm{P}-1$ & 4,1 & $-1,0$ \\
\hline P-2 & 3,1 & 0,3 \\
\hline$P-3$ & 2,8 & 0,4 \\
\hline P. 4 & 2,7 & 0,7 \\
\hline$P-5$ & 2,5 & 0.7 \\
\hline$P-6$ & 2,4 & 1,0 \\
\hline$P-7$ & 2,3 & 0,9 \\
\hline$P \ldots 8$ & 1,8 & 1,7 \\
\hline
\end{tabular}

A reta de correlação 8018 versus $\$ C 13$ obtida para os carbonatos das conchas que se desenvolvem em pontos de salinidades diversas dentro da zona de mistura, pode ser utilizada para verificar se a formação destes organismos efeluou-sc em equilíbrio isolópico com as espécies carbonaladas dissolvidas. Assim, extrapolando a rela de correlação acima para o valor $\$ 018-4,8 \%$. (PDB) (carbonato formado em água de salinidade $0 \%$.) encontra-se um valor de $8 \mathrm{C}$ 13 - $-2,0 \%$. (PDB). Como a razão C 13/C 12 dos biocarbonafos dissolvidos na água doce apresenta um valor médio $8 \mathrm{C} 13$ $-11,3 \%$. (PDB), (Pina, 1976), pode-se concluir que os carbonalus clas cunchas não são formados em equilíbrio isotópico com o meio ambiente, pelo menos no que concerne os isótopos do carbono.

No que diz respeito ao comportamento dos isótopos do oxigênio, McCrea (1950), Epstein et al $(1951,1953)$ mostraram que estes poderiam ser utilizados como indicadores da chamada termoetria isotópica dos carbonatos, ou seja, a composição isotópica O 18/O 16 resultaria de partições inorgânicas e biológicas dos isólopos de oxigênio, envolvendo $\mathrm{CaCO} 3 \mathrm{e}$ H20 em função da temperatura.

Por outro lado, Peixinho (op. cit.), ao desenvolver estudos no estuário do rio Paraguaçu, mostrou que a temperatura média das águas era de aproximadamente $28^{\circ} \mathrm{C}$. Fazendo uso da relação $\$ O 18$ (água) $=-4,2+0,124 \mathrm{~S}(\%$.), obtida através do gráfico de $S(\%$.) versus $\$ 018($ SMOW) da água (Figura 2), o valor do $\$ O 18(\mathrm{PDB})$ para carbonatos formados nessa água pode ser determinado a partir da equação de Craig (1965):

$\left({ }^{\circ} \mathrm{C}\right)=16,9-4,2(8 \mathrm{O} 18-\mathrm{A})+0,13($ \$ $18-\mathrm{A}) 2$

SO $18=\$ 018$ do carbonato de cálcio formado em equilíbrio (\%.) $\quad A=8018$ da solução (\%.).

A equação (1) resulta em:

$28-16,9=-4,2 \mathrm{~V}+0,13 \mathrm{~V} 2$, onde $\mathrm{V}=\$ \mathrm{O} 18(\mathrm{CaCO} 3)=$ \$O 18 (água)

Resolvendo para $\mathrm{V}$, obtem-se:

$(\mathrm{V}-13,3)(\mathrm{V}+2,2)=0$, onde $\mathrm{V}=8 \mathrm{O} 18(\mathrm{CaCO} 3)-8 \mathrm{O}$ 18 (água) $=-2,2$

$8018(\operatorname{CaCO} 3)=-6,4+0,124 \mathrm{~S}(\%$.

Como para cada amostra de carbonato é conhecido o valor da composição isotópica do oxigênio é possível pois determina a salinidade média local durante o crescimento sazonal da concha. Os resultados obtidos são apresentados na Tabela 4.

\section{TABELL 4}

\section{(a) Resultados da sallinidade médla local do crescimento de conchas carbonatadas segundo equação (2)}

\begin{tabular}{|c|c|}
\hline AMOSTRA & $S \mid \%(\%)$ \\
\hline P 1 & 26. \\
\hline P2 & 28,8 \\
\hline P3 & 30,2 \\
\hline P-4 & 31,1 \\
\hline$p .5$ & 26,4 \\
\hline $\begin{array}{l}P 6 \\
0.7\end{array}$ & $\frac{32,5}{323}$ \\
\hline$\frac{P-7}{P-8}$ & $\frac{33.2}{372}$ \\
\hline
\end{tabular}

\section{(b) Resultados da salinidade médla anual de alguns pontos da bacia do rio Paraguaçu determinados Segundo Peixinho (1974)}

\begin{tabular}{|c|c|}
\hline ESTAC̣AOO & $S\left|\%_{0}\right|$ \\
\hline 1 & 26,6 \\
\hline 2 & 29,0 \\
\hline 3 & 28,8 \\
\hline 4 & 29,0 \\
\hline \multicolumn{2}{|c|}{$\begin{array}{l}\text { OBS.: } \\
\text { As estaçós } 1,2,3 \text { e } 4 \text { estão situadas proximas } \\
\text { dos pontos } P-2 ; P-5 ; \text { entre } P-2 \text { \& } P-3 ; \text { \& } P-4 \\
\text { respectivamente. }\end{array}$} \\
\hline
\end{tabular}


A comparação dos valores calculados para as salinidades a partir das relações deduzidas acima com aqueles obtidos por Peixinho (op. cit.), mostram uma boa concordância, o que sugere que os carbonatos ter-se-iam formado em equilíbrio cum o oxigênio da água.

\section{CONCLUSÕES}

Os resultados obtidos mostran que:

1. Há uma forte correlação entre salinidade e composição isolópica da água na zona de mistura do rio Paraguaçu com a Baía de Todos os Santos, o que indica que ocorre uma mislura conservativa entre a água do mar e água do rio;

2. A razão isotópica $O 18 / 016$ média das águas nesta zona é representativa dos valores isotópicos das precipitações na região:

3. Verifica-se a existência de correlação linear entre $\$ C 13$ e 8018 dos carbonatos, porém os valores do \$C 13 para os bicarbonalos dissolvidos não são compatíveis com os valores isotópicos do carbonato, possivelmente por não haver equilíbrio para o carbono na deposição do carbonato;

4. Utilizando-se as razões isotópicas $O 18 / 016$ dos carbonatos para calcular a salinidade média, verifica-se boa concordância com valores médios anuais da salinidade determinada na região, o que permite admitir que a formação da concha se deu em equilíbrio isotópico com o oxigênio da água.

\section{AGRADECIMENTOS}

$O$ autor agradece aos Drs. Robert E. Sweeney e Jean Marie Flexor pelas crílicas e sugestões na realização desse trabalho, bem como às entidades financiadoras FINEP, BNDE e CNPq, alravés do Programa de Pesquisa e Pós-Graduaçãu em Geofísica da Universidade Federal da Bahia.

\section{BIBLIOGRAFIA}

AZEVEDO A.E.G. (1975) - Relatório Interno PPPG/UFBa.

CAMPOS, H.S. (1976) - Estudo das vatiaçóes isotópicas C 13/C 12 e $\mathrm{O}$ 18. ${ }^{\circ} \mathrm{O} 16 \mathrm{em}$ ambientes de formaça de rochas de praia na liha de Itaparica. Bahlia. Tese de Mestrado do PPPG/UFBA.

COMPANHIA DE PESQUISA E RECURSOS MINERAIS (1975) - Relalírios mensais enviados à Secretaria de Recursos Hídricos do Estado da Ballia, Salvader, Brasil.

CRAIG, H. (1965) - The measurement of oxygen isotope paleotemperatures. Proc. Spoleto Conf. on Stable isotopes in oceanografic studies and palleotemperal lures, Pisa.

DANSGAARD, W. (1961) - The isotopic composition of natural waters. Meddeletser on Gronland 165,1

EPSTEIN, S. A MAYEDA, T. (1953) - Variations of O 18 conten! of waters from natural sources. Geochim. el Comochim. Acta 4,213.
EPSTEIN, S., BUCHSBAUM, R., LOWENSTAM, H.A. E UREY, H. C. (1951) - Carbonate-water isotopic temperature scale. Bull. Geol.Soc. Am. 62, 417.

MCCREA, J.M. (1950) - On the isotopic chemistry of carbonates and a paleotemperature scale. J. Chem. Phys. 18, 849.

MOOK, W.G. (1968) - Geochemistry of the stable carbon and oxygen iso(o)es of natural waters in the Netherlands. $\mathrm{Ph} . \mathrm{D}$, Thesis Rijkstuniversiteitte Groninger. pp. 157.

PEIXINHO, V. (1974) - Relatório semestral enviado ao Conselho Nacional de Pesquisa (CN\$q) - Rio de Janeiro, RJ.

PINA, L.C. (1976) - Estudo do comportamento dos elementos C, O, Ca, Mg no estuário do rio Paraguaçu-Bahia. Tese de Mestrado do PPPG/ UFBa, 